\title{
EXPERIMENTAL INVESTIGATION OF A NEWLY DESIGNED SUPERSONIC WIND TUNNEL
}

\author{
J. Wu and R. Radespiel \\ Institute of Fluid Mechanics \\ Technische Universität Braunschweig \\ 37 Herman-Blenk-Str., Braunschweig 38108, Germany
}

\begin{abstract}
The flow characteristics of the tandem nozzle supersonic wind tunnel at the Institute of Fluid Mechanics, Technische Universität Braunschweig, are investigated. Conventional measurement techniques were utilized. The flow development is examined by pressure sensors installed at various streamwise positions. The temperature is measured in the storage tube and the settling chamber. The influence of flow treatment in the settling chamber on the flow quality is also studied. The flow quality of test section is evaluated by a 6 -probe Pitot rake. The pressure fluctuations in the test section are studied by a sharp cone model. Eventually, good agreement between the measurements and numerical simulation of the tunnel design is achieved.
\end{abstract}

\section{INTRODUCTION}

Supersonic flow research is an important field for applications such as supersonic cruising aircraft, space launchers, and reentry vehicles. Fundamental studies of supersonic flow are particularly needed in areas of large prediction uncertainties, particularly, in shock wave-boundary layer interactions, afterbody aerodynamics at high Reynolds numbers, and laminar-turbulent transition on wings, blunt bodies, and slender bodies. As the uncertainties in these fields are mainly caused by the lack of reliable numerical flow models, wind tunnel experiments are presently the major source of knowledge in these areas. In supersonic wind tunnels, the Ludwieg tube principle offers an inherent low disturbance flow environment suited for boundary-layer studies due to its mode of operation [1]. However, in its simplest setup with a fixed nozzle, a Ludwieg tube can only represent a single Mach number in the test section. With exchangeable single nozzle, only a very limited range of Mach numbers is possible that cannot cover both supersonic and hypersonic flow ranges. This limitation can be overcome by

This is an Open Access article distributed under the terms of the Creative Commons Attribution License 4.0, which permits unrestricted use, distribution, and reproduction in any medium, provided the original work is properly cited. 


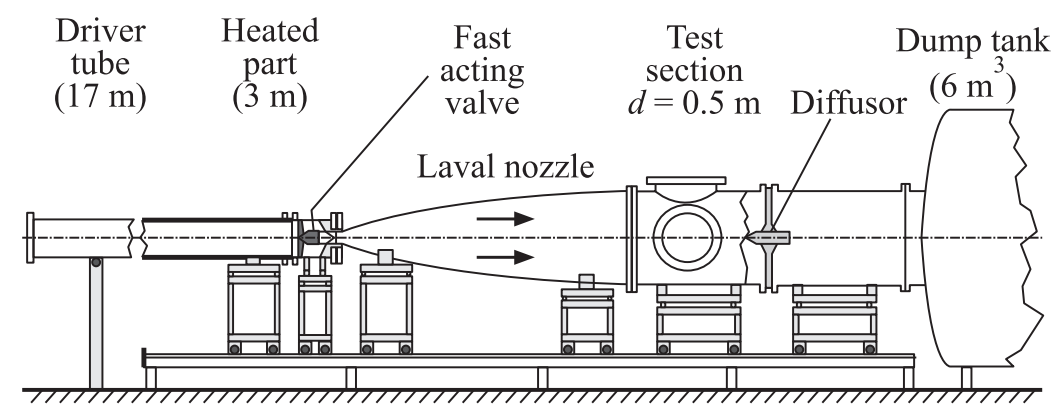

Figure 1 Sketch of the HLB

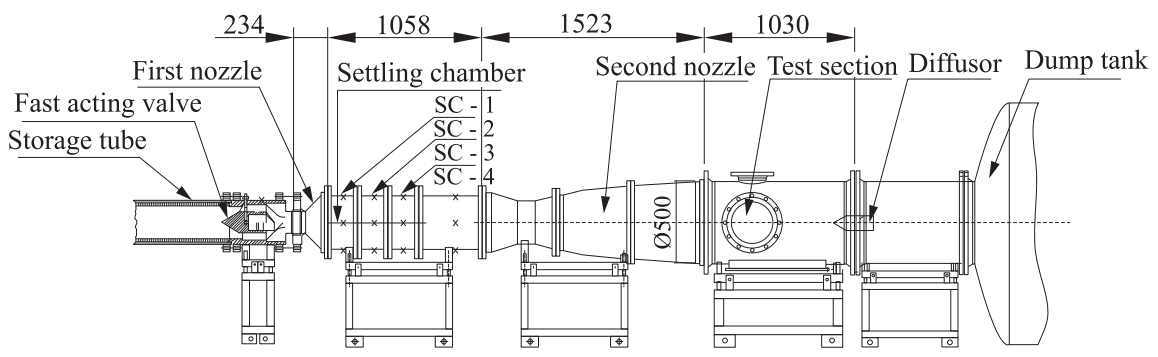

Figure 2 Sketch of tandem nozzle supersonic wind tunnel. Dimensions are in millimeters

introducing the tandem nozzle [2]. Following this approach, an axisymmetrical Mach 3 tandem nozzle supersonic wind tunnel was designed at the Institute of Fluid Mechanics, Technische Universität Braunschweig [3]. The tandem nozzle supersonic wind tunnel was designed based on the infrastructure of the existing Hypersonic Ludwieg Tube of Braunschweig (HLB) [4], as shown in Fig. 1, which can produce a Mach 6 hypersonic flow for $80 \mathrm{~ms}$ and reach unit Reynolds numbers up to $20 \cdot 10^{6}$. The tandem nozzle supersonic wind tunnel alters the original Mach 6 flow to a Mach 3 flow using two nozzles and an additional settling chamber, according to Fig. 2, while it retains the test section size and the tunnel running time.

Due to the introduction of the newly added nozzle and settling chamber, the flow inside undergoes changes during the tunnel run. According to computational fluid dynamics (CFD) simulations [3], strong flow separations occur in the first nozzle because of the shock/boundary-layer interactions. Hence, it is crucial to make an overall assessment of the flow quality in the wind tunnel. The assessment of the tandem nozzle tunnel with dedicated measurements is, therefore, the subject of the present paper. Conventional measurement 
techniques are utilized for this purpose. The static pressure of storage tube and settling chamber is measured by pressure sensors, which were installed in the storage tube and settling chamber. These measurements provide an impression of how the flow rebuilds before reaching the second nozzle. In order to evaluate the temperature distribution, three thermocouples were placed around the central valve body ahead of the first nozzle with an angular offset of $120^{\circ}$ (central axis) and four thermocouples were installed at the rear part of the settling chamber with an angular offset of $90^{\circ}$ (central axis) around the wall. Quantitative data of the flow field in the test section were obtained using a 6-probe Pitot rake that was traversed both in streamwise and traverse directions. Through the Pitot rake measurements, the flow uniformity of the entire test section can be evaluated. Special attention is paid to the quantitative influence of the settling chamber configuration to the flow quality of the test section. Perforated plates and screens are installed in the settling chamber in different configurations. The flow quality is inspected by measuring the static and dynamic pressure responses in the test section. As a result, an optimum combination of perforated plates and screen is obtained. Furthermore, a preliminary assessment of unsteady free-stream flow disturbances is provided by investigating pressure disturbances and boundary-layer transition on a 7 degree half-angle sharp cone model with flush mounted fast response pressure sensors.

\section{EXPERIMENTAL APPARATUS}

\subsection{Working Principle}

The working mechanism of the tandem nozzle supersonic wind tunnel differs from the traditional Ludwieg tube, which consists of a storage tube, a nozzle, a test section, and a dump tank [1]. Depicted in Fig. 2 is a schematic diagram of the tandem nozzle supersonic wind tunnel. A new nozzle and settling chamber are added based on the Ludwieg tube. This modification of the design can alter the flow Mach number without changing the main infrastructure of the tunnel, which saves considerable cost and time.

The tandem nozzle supersonic wind tunnel works as follows. The highpressure and high-temperature air is contained in the storage tube. The storage tube is separated from the first supersonic nozzle by a fast-acting valve. As the valve opens, expansion waves $(O A$ and $O B$ in Fig. 3$)$ travel upstream into the storage tube and are reflected $(A C$ and $B D)$ when the flow reaches the end of storage tube (see Fig. 3). This expansion provides a quasi-constant pressure and temperature, which are treated as the stagnation pressure and stagnation temperature during the tunnel operation. Meanwhile, the air expands into the first 


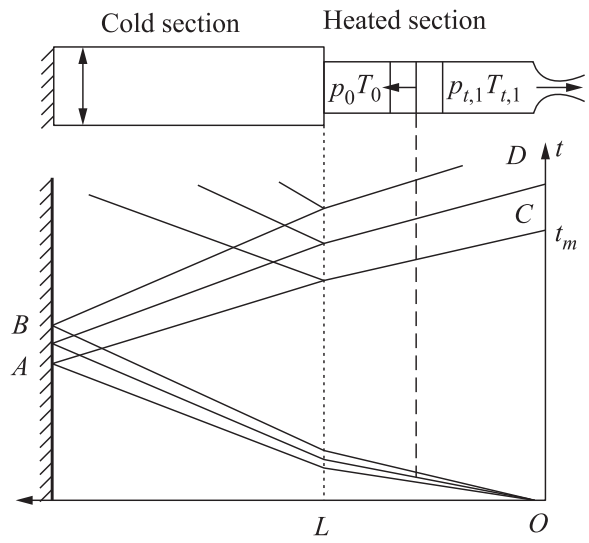

Figure 3 Operation principle of the Braunschweig Ludwieg tube

nozzle with a sonic throat. Further downstream, a system of shocks develops within the first nozzle, due to the pressure imposed by the fixed mass flow of the second nozzle. These shocks in the first nozzle also cause flow separation due to boundary-layer interaction. The flow reestablishes then into an organized state within the settling chamber. Using several damping devices to settle the flow, good flow uniformity can be obtained before the flow enters the second throat. Finally, the flow accelerates along the second nozzle and achieves the desired supersonic state in the test section. The tunnel will stop running when the reflected expansion wave reaches the first throat again. More details about the tandem nozzle principle can be found in $[3,5]$.

During the operation of the wind tunnel, the total pressure undergoes variations after the expansion wave in the storage tube. Assume the initial pressure and temperature in hot section before the tunnel running are $T_{0}$ and $p_{0}$, and the stagnation temperature and pressure after the expansion wave are $T_{t, 1}$ and $p_{t, 1}$. Then, the following ratios can be obtained [5]:

$$
\begin{aligned}
\frac{T_{t, 1}}{T_{0}} & =\frac{1+((\gamma-1) / 2) \mathrm{Ma}^{2}}{(1+((\gamma-1) / 2) \mathrm{Ma})^{2}} \\
\frac{p_{t, 1}}{p_{0}} & =\left[\frac{1+((\gamma-1) / 2) \mathrm{Ma}^{2}}{(1+((\gamma-1) / 2) \mathrm{Ma})^{2}}\right]^{\gamma /(\gamma-1)}
\end{aligned}
$$

where $\mathrm{Ma}$ is the Mach number in the storage tube and $\gamma$ is the ratio of the specific heats for a perfect gas.

Since the mass flow for the first throat and for the second throat are the same when sonic flow conditions are assumed, then total pressure of the second throat is given by $[6]$ : 


$$
\frac{p_{t, 1 \mathrm{st}}}{p_{t, 2 \mathrm{nd}}}=\left(\frac{D_{2 \mathrm{nd}}}{D_{1 \mathrm{st}}}\right)^{2}
$$

where $D$ is the diameter of the cross section of throat, and subscript 1st denotes the first throat while 2nd refers to the second throat. The difference of total pressure between the two throats is yielded by the shock interactions and the settling chamber viscous loss.

\section{EXPERIMENTAL METHODS}

\subsection{Pressure Measurement}

Three types of transient pressure measurements with medium temporal resolution were performed. The first was the pressure measurement in the storage tube. The pressure sensor was installed at the valve mounting. This pressure measurement provides the development of stagnation pressure from the storage tube. The second pressure measurement was conducted in the settling chamber. Sixteen Honeywell TruStability Standard Accuracy Silicon Ceramic (SSC) pressure sensors were installed in the settling chamber and where each measurement section had 4 sensors located with a circumferential angle offset of $90^{\circ}$, as marked by ' $x$ ' shown in Fig. 2. This measurement was aimed at evaluating the pressure drop of the damping devices in set-

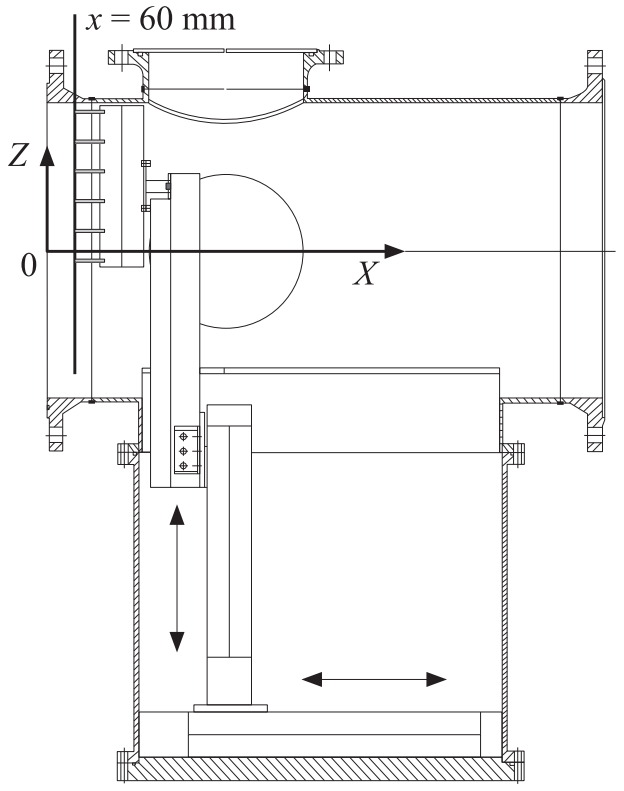

Figure 4 Sketch of Pitot rake traversing in test section

tling chamber. The third type of pressure measurement was carried out in test section. A 6-probe Pitot rake was employed. The Pitot rake can traverse in both $x$-(streamwise) and $z$-(vertical) directions driven by an electrical stepper motor. The overall Pitot rake measurement covered a streamwise distance of $350 \mathrm{~mm}$ and a vertical distance of $400 \mathrm{~mm}$. The setup is shown in Fig. 4. Furthermore, the Mach contour of a cross section along streamwise direction was measured by traversing the Pitot rake in both $y$-(horizontal) and $z$-(vertical) directions. The 
sensors of the Pitot rake are RMO (Reverse Mode of Operation) series sensors from Sensortechnics.

The experimental data were acquired by a Spectrum M2i.4652 transient recorder with a maximum sampling rate of $3 \mathrm{MHz}$. In these transient pressure measurements, the sampling rates of RMO sensors and SSC type sensor were set to be 10 and $1 \mathrm{kHz}$, respectively.

\subsection{Temperature Measurement}

Temperatures were measured using Omega Nickel-Chrome/Nickel thermocouples (K type) in the storage tube and the rear part of the settling chamber. The thermocouple wire has a diameter of $0.04 \mathrm{~mm}$ and a dynamic response about $500 \mathrm{~Hz}$. Three thermocouples were installed around the central valve body with an angularity offset of $120^{\circ}$ with respect to the central axis, as shown in Fig. $5 a$. These thermocouples measure the temperature of flow before it enters the first nozzle. The flow in the storage tube of HLB has a quite low velocity and large density; therefore, the thermocouple surface obtains a high recovery temperature of almost the total temperature [4]. The temperature for the inlet flow of the second nozzle was measured at the last section of the settling chamber. Four thermocouples were installed separately on the top, bottom, right, and left positions of the settling chamber, as shown in Fig. $5 b$. The thermocouples were mounted via a thin steel pipe inserting into the flow. The tip of the pipe with the active sensor wire was about $95 \mathrm{~mm}$ away from the internal wall towards the central axis.

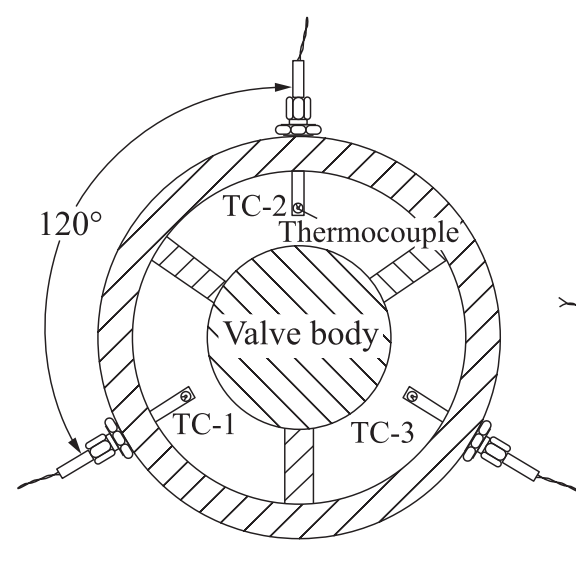

(a)

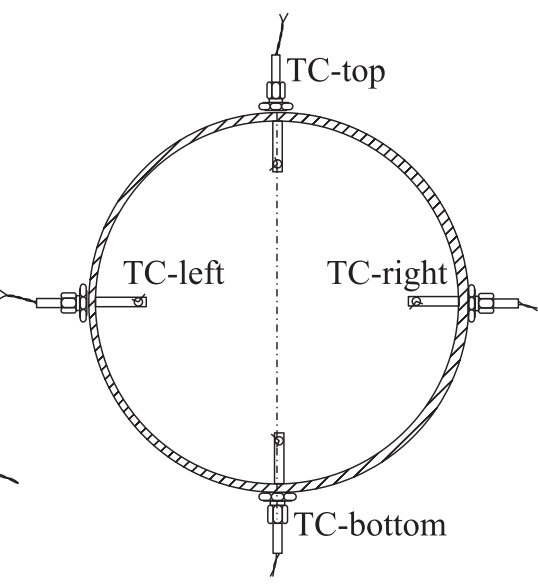

(b)

Figure 5 Sketch of temperature measurement locations: thermocouples on the storage tube $(a)$ and in the settling chamber $(b)$ 


\subsection{Sharp Cone Measurement}

Dynamic measurements in the wind tunnel were accomplished by PCBM132A31 sensors installed on a 7 degree half-angle sharp cone model [7]. The PCB sensors are flush mounted to measure the surface pressure fluctuation. This sensor has a diameter of $3.18 \mathrm{~mm}$ and a quadratic active area of $0.768 \times 0.768 \mathrm{~mm}$. It is used in pressure fluctuations measurement due to its broad dynamic response range, which is from $10 \mathrm{kHz}$ to $1 \mathrm{MHz}[8,9]$. The power for the PCB sensor was supplied by two PCB instruments (M482A22 and M483A). The tip of the model is made of stainless steel with a bluntness of about $100 \mu \mathrm{m}$ in diameter. The cone is comprised of 11 parts and has a total length of $400 \mathrm{~mm}$ as shown in Fig. $6 a$. It permits the installation of sensors at 11 different locations along the cone body. In the present study, $10 \mathrm{PCB}$ sensors were used. These sensors were divided into three groups. The first group included the first two sensors located at $x=209$ and $234 \mathrm{~mm}$, respectively. The second group, marked by G1 in Fig. 6, had four sensors, three sensors of which were installed in a circumferential array while one sensor was installed $6 \mathrm{~mm}$ ahead. The third group was $100 \mathrm{~mm}$ downstream of the second group sensor and it had a similar distribution with the second group sensor, as shown by G2 in Fig. $6 a$. The detailed dimensions of the cone model and the sensor locations are provided by Fig. $6 b$. The data acquisition card is the Spectrum M2i.4652 transient recorder, and the sampling frequency was set to $2.5 \mathrm{MHz}$ in the sharp cone measurement.

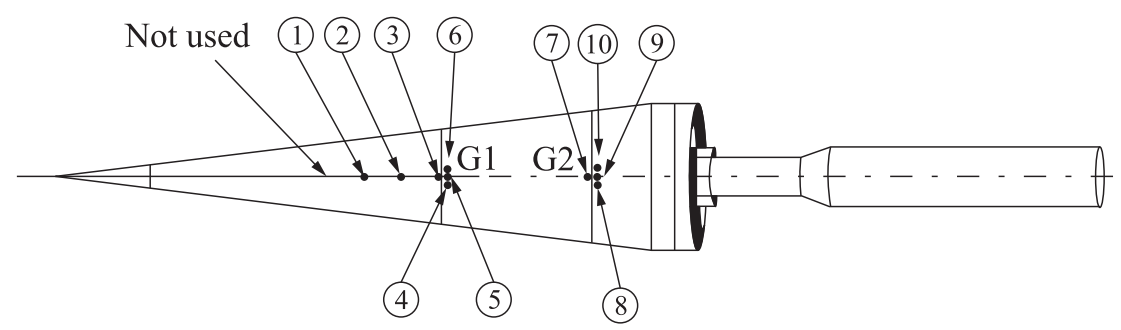

(a)

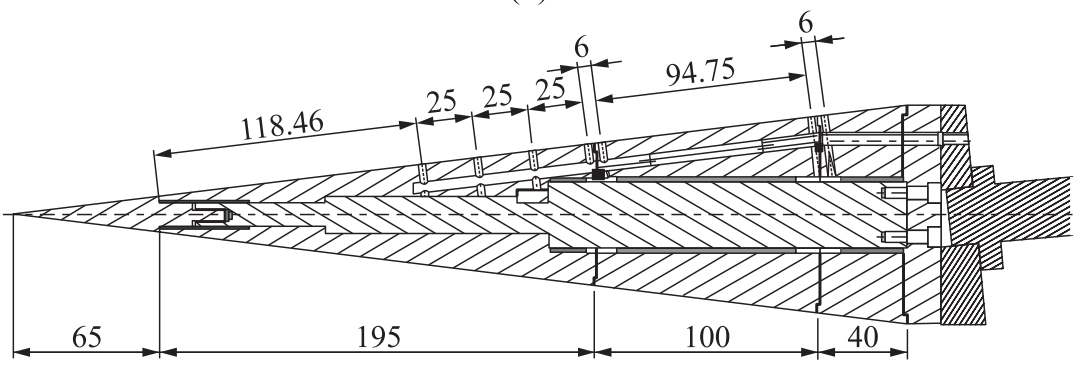

(b)

Figure 6 Cone model used in the experiments [7]. Dimensions are in millimeters 


\subsection{Data Analysis}

The signals of the PCB sensors are evaluated by Fourier transform based on a 50-millisecond experimental duration, which is divided into segments of 1024 data points, with an overlap of 768 points. Each window is multiplied by a normalized Blackman window and Fourier transformed. This results in a frequency resolution of $2.9 \mathrm{KHz}$. Afterwards, the spectra are averaged. Note that the final spectra of pressure are obtained by subtracting the amplitude of signals before the tunnel run to reduce the electric noise.

The Pitot rake measurements in the test section are evaluated in the form of Mach number. The total pressure ahead of the second nozzle can be calculated based on the measured static pressure and the Mach number in the last section of the settling chamber. Using the well-known Rayleigh-Pitot formula, the Mach number is obtained:

$$
\frac{p_{\text {pitot }}}{p_{t, 2 n d}}=\left[\frac{\gamma+1}{2 \gamma \mathrm{Ma}^{2}-(\gamma-1)}\right]^{1 /(\gamma-1)}\left[\frac{(\gamma+1) \mathrm{Ma}^{2}}{(\gamma-1) \mathrm{Ma}^{2}+2}\right]^{\gamma /(\gamma-1)}
$$

where $p_{\text {pitot }}$ is the pressure measured by the Pitot probe.

\section{EXPERIMENTAL RESULTS}

\subsection{Pressure Distributions Along Wind Tunnel}

The pressure distribution along the wind tunnel is important in the design of tandem nozzle tunnel, because it provides information of how flow develops inside the tunnel. This helps to understand the working mechanism of the tandem nozzle type tunnel better. First, let check whether both throats satisfy the assumption of sonic flow. Otherwise, a desired Mach number would not be available in the test section. Thus, the total pressure of the second throat has to be checked. Figure 7 displays pressure transients at various streamwise positions for one tunnel run. Here, four perforated plates were installed in the settling chamber (see subsection 4.3).

'Valve' in the legend of Fig. 7 is the time trace of static pressure at the fast acting valve; 'SC-1' to 'SC-4' are the static pressure transients measured at each section of the settling chamber (see Fig. 2); and 'pitot-probe' is the pressure measurement of Pitot probe at the position of $x=60 \mathrm{~mm}$ and $z=0 \mathrm{~mm}$ in the test section. This signal shows that the tandem nozzle tunnel has an effective measurement time of about $60 \mathrm{~ms}$. The total pressure drop between the first nozzle and just behind the first perforated plate takes up most of the total pressure loss. The result of the pressure drop agrees well with the numerical design in [2], which demonstrates that the perforated plates mainly provide flow 


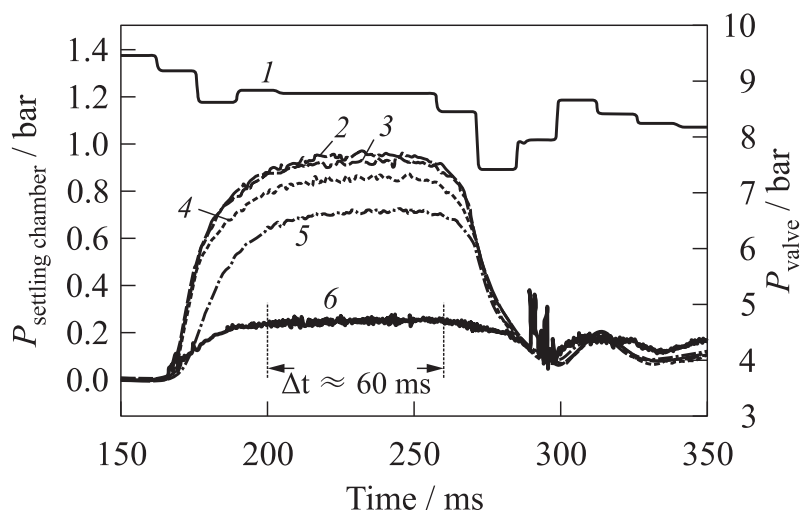

Figure 7 Pressure distribution in tandem nozzle wind tunnel, $P_{t, 1}=9.5$ bar, $T_{t, 1}$ $=430 \mathrm{~K}: 1-$ valve; $2-\mathrm{SC}-1 ; 3-\mathrm{SC}-2 ; 4-\mathrm{SC}-3 ; 5-\mathrm{SC}-4$; and $6-$ pilot-probe

quality improvement. The fourth perforated plate exhibits the largest pressure drop among all the plates in the settling chamber. According to Eq. (1), the total pressure of the second throat can be calculated as 0.763 bar. Also, the total pressure of the second throat can be calculated based on the static pressure and Mach number in the last section of settling chamber, since flow from this last section to the test section is considered as isentropic flow. The static pressure is known from Fig. 7, and the Mach number at that section can be obtained by the Area-Mach number relation [5]:

$$
\frac{A}{A^{*}}=\frac{1}{\mathrm{Ma}^{2}}\left(\frac{2}{\gamma+1}\left(1+\frac{\gamma-1}{2} \mathrm{Ma}^{2}\right)\right)^{(\gamma+1) /(\gamma-1)}
$$

where $A$ is the area and asterisk symbol means the sonic critical condition. Eventually, the total pressure of the second throat is 0.759 bar, which means a 0.56 percent difference to the value calculated by Eq. (1). Thus, sonic flows are present in both throats.

\subsection{Temperature Distributions in Wind Tunnel}

The positions of the thermocouples are indicated in Fig. 5. The temperatures in front of the first throat were measured both in cold and hot tunnel runs (Fig. 8); TC-1, TC-2 and TC-3 are the temperature variations of thermocouples marked in Fig. $5 a$. The upper thermocouple (TC-2) always shows a higher temperature than the lower thermocouples. However, the extent is much less in cold tunnel run, while in heated operation, the maximum temperature difference can reach 

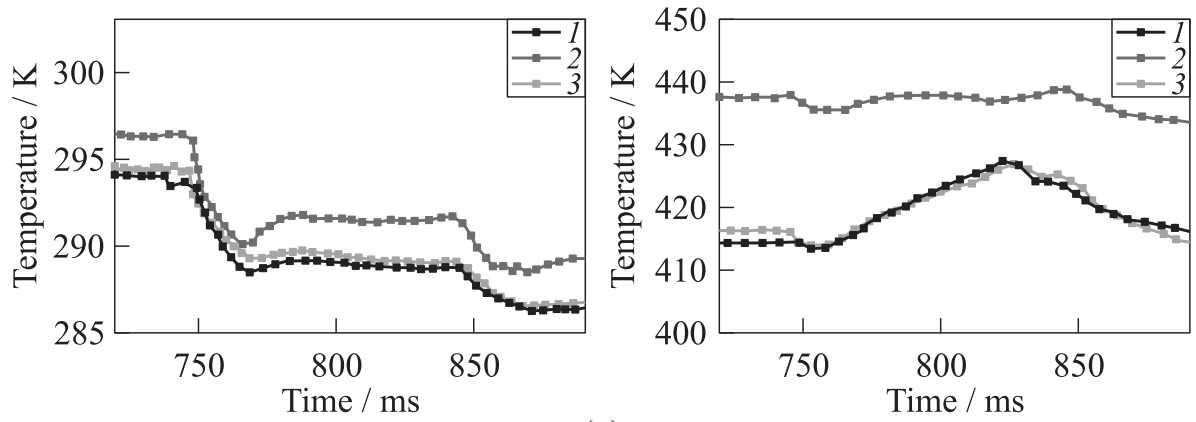

(a)
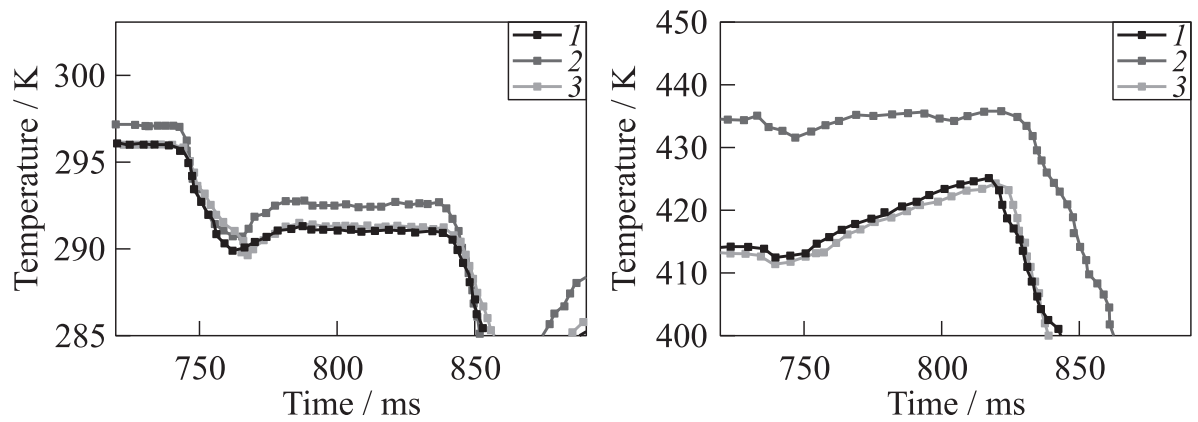

(b)
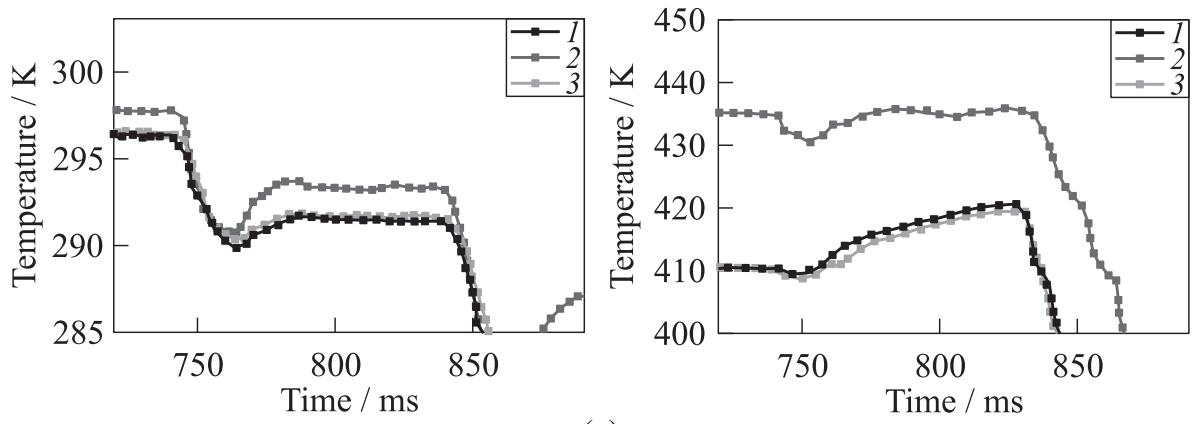

(c)

Figure 8 Variations of temperatures in front of the first throat $(1-$ TC-1;2 TC-2; and $3-$ TC-3) in both cold and hot tunnel runs (left column $-T_{0}=300 \mathrm{~K}$ and right column $\left.-T_{0}=430 \mathrm{~K}\right):(a) p_{0}=10 \mathrm{bar} ;(b) 15$; and $(c) p_{0}=20 \mathrm{bar}$ 


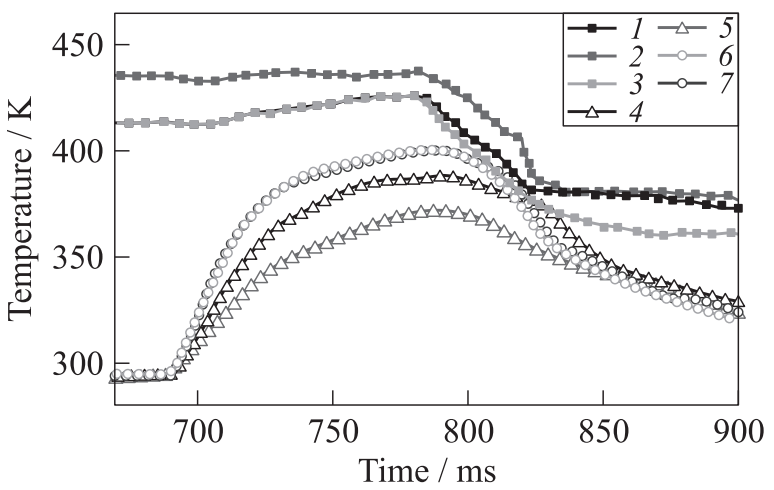

Figure 9 Temperature transients in tandem nozzle supersonic wind tunnel; $p_{0}$ $=10$ bar, $T_{0}=430 \mathrm{~K} ; 1-$ TC- $1 ; 2-$ TC-2; 3 - TC-3; 4 - TC-right; 5 TC-bottom; 6 - TC-left; and 7 - TC-top

approximately $25^{\circ}$. The differences of the temperature measured in both cold and heated tunnel runs indicate air stratification in the storage tube, and this phenomenon is stronger at higher temperatures in the storage tube. Moreover, the measurements show that the cold runs yield a longer constant time trace compared to the heated runs.

The aforementioned measurement is the temperature before the first throat which is less important compared with the temperature in front of the second nozzle. Because of the flow separations in the first nozzle and the installation of perforated plates in the settling chamber, the flow total temperature undergoes a significant change from the first nozzle to the last section of the settling chamber. Preliminary measurements indicated that the individual thermocouples at the different positions of Fig. $5 b$ show a different dynamic response to transient flow behavior. In order to eliminate the dynamic response for different thermocouples, one thermocouple was chosen and placed at different positions around the settling chamber. The results of these tests are shown in Fig. 9.

It appears that flow in the last section of the settling chamber is not fully axisymmetrical. The flow at the bottom of the settling chamber has the lowest temperature due to the effect of storage tube stratification. The temperature of the right side is slightly lower than the temperatures of the top and left positions. As the dynamic response of the sensor itself depends on flow density, additional measurements were performed at different storage tube pressures while the storage tube temperature was kept constant. The corresponding thermocouple transients of TC-right are displayed in Fig. 10.

It is seen that the thermal response of the thermocouple is somewhat faster at higher pressures indicating that the sensor is quite at its physical limit to follow the flow temperature variation, at the flow conditions of the settling chamber. 


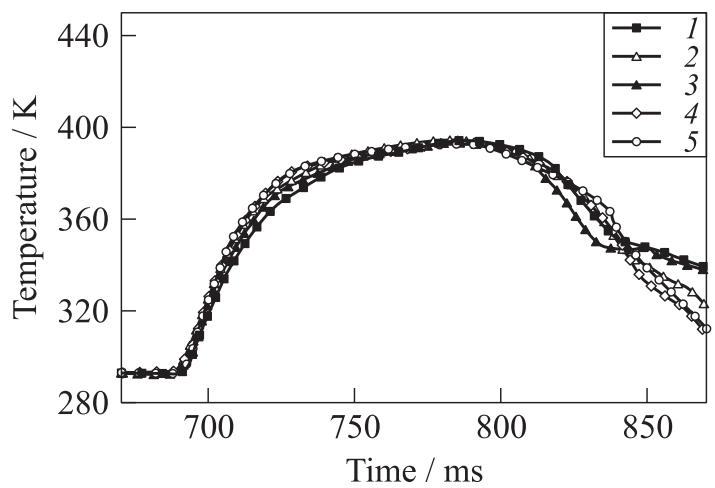

Figure 10 Temperature transient comparison of different storage pressures at TCright position; $T_{0}=430 \mathrm{~K}: 1-10 \mathrm{bar}, 2-12.5 ; 3-15 ; 4-17.5$; and $5-20$ bar

However, the temperature signals appear to converge towards the storage pressure of 20 bar. This indicates that the physical temperature variation in time for heated conditions during the useful run time of the facility $(0.73-0.78 \mathrm{~s})$ is about $10 \mathrm{~K}$. This variation could be caused by the spatial variation of storage tube stratification (see readings of TC- 1 and TC 3 in Fig. 9) and by the heat transfer in the perforated plates.

\subsection{Settling Chamber Measurement}

The requirement for a homogeneous flow in the test section is a uniform incoming flow from the settling chamber, which is determined by the settling chamber design. Designing the settling chamber appears as making a compromise between good flow quality and achieving a long tunnel running time. Using more devices, the flow quality is better while the flow needs more time to settle in the settling chamber, whereas too few damping devices cannot provide a uniform flow. Steady axisymmetrical Reynolds-averaged Navier-Stokes (RANS) simulations of the effect of perforated plates on flow quality [3] show an optimal number of perforated plates of about 3-4. Note that the use of screens was not investigated by numerical flow simulations. A screen is known to reduce the length scale of turbulence and this is used frequently in wind tunnels. However, a reliable numerical simulation of screens at high-Reynolds numbers is not possible and only empirical correlations exist for their effect on disturbance velocities [10]. Therefore, an experimental investigation is conducted by replacing the perforated plates with screens and recording static pressure in the settling chamber and the Pitot rake signals in the test section. The perforated plates are made 
from aluminum (EN AW-2007) with holes in an equilateral triangular pattern. Two different open ratios are used for the perforated plates. The first perforated plate is located at the end of the first nozzle, which needs to stand strong forces generated by the unsteady flow build up. Therefore, a larger thickness of $20 \mathrm{~mm}$ and an open ratio of $65 \%$ is defined for the first perforated plate, while other plates have a smaller open ratio of $41 \%$ and a smaller thickness of $17 \mathrm{~mm}$. The axial distance between the plates is $250 \mathrm{~mm}$.

The effect of the perforated plates on the static pressure transients is displayed in Fig. 11. With four perforated plates installed in the settling chamber, the static pressure close to the second nozzle obtains the smallest oscillations. Therefore, four perforated plates are used as a basic configuration in further measurements. Here, representative values of the pressure are obtained by taking averages between $t=0.22$ and $0.24 \mathrm{~s}$. The pressures along the settling cham-

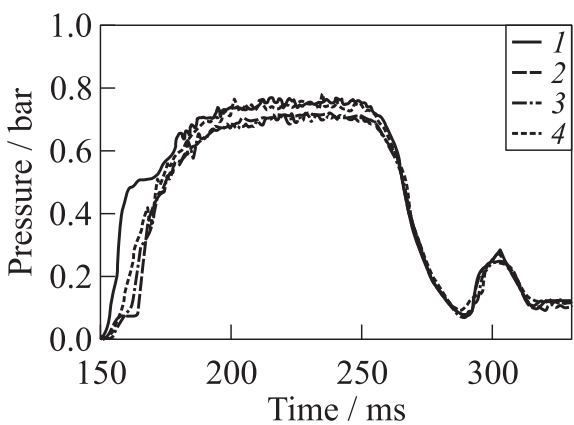

(a)

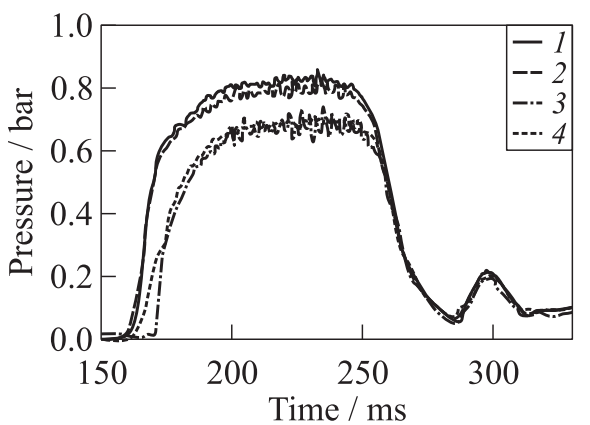

(b)

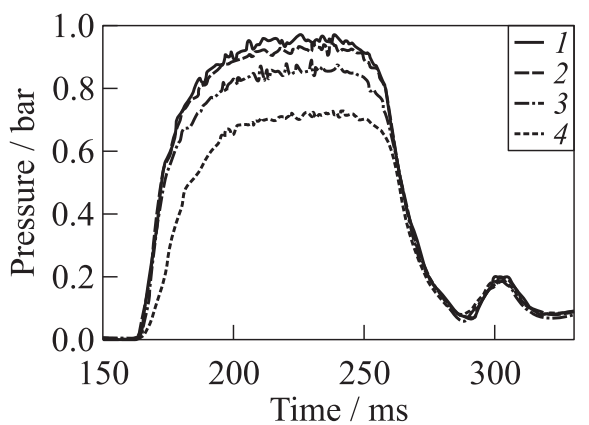

(c)

Figure 11 Static pressure transients $(1-\mathrm{SC}-1 ; 2-\mathrm{SC}-2 ; 3-\mathrm{SC}-3$; and $4-$ SC-4) in the settling chamber for different numbers of perforated plates $\left(P_{0}=10\right.$ bar and $T_{0}=300 \mathrm{~K}$ ): (a) 2 perforated plates; (b) 3 perforated plates; and (c) 4 perforated plates 


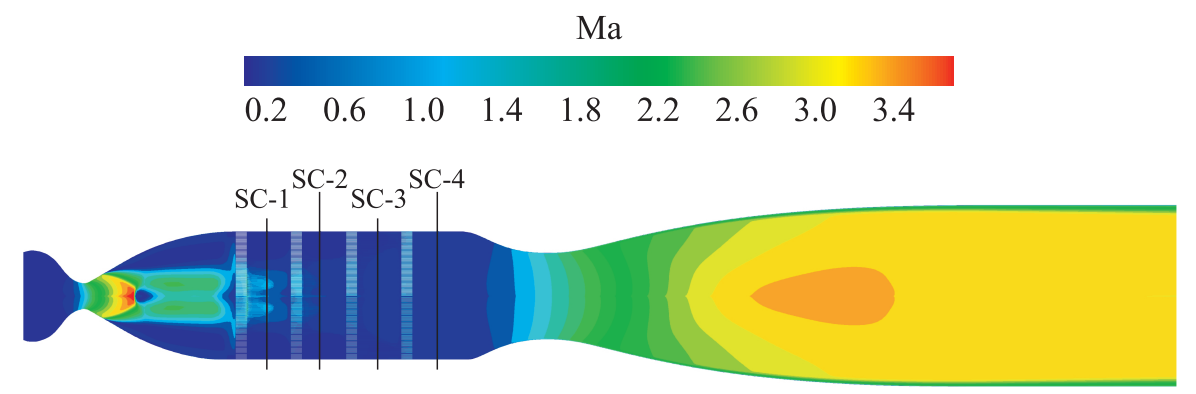

(a)

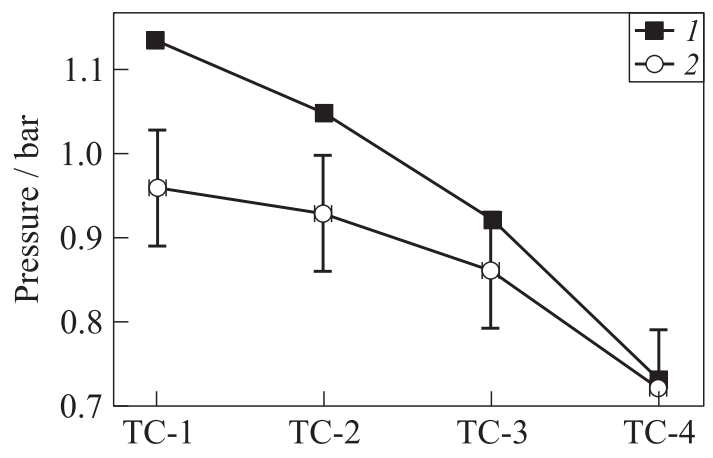

(b)

Figure 12 Comparison of numerical and experimental flow data in the settling chamber: (a) Mach number contour of RANS simulation of the tandem nozzle supersonic wind tunnel; and $(b)$ pressure distribution comparison along the settling chamber wall $\left(1\right.$ - numerical simulation; and 2 - experimental measurement, $\left.P_{\text {storage }}=10 \mathrm{bar}\right)$

ber wall from numerical simulation data are extracted and compared with the experimental result, which is shown in Fig. 12. The present authors observed that the pressure drop was smaller than predicted by the RANS simulation. This discrepancy might be caused by the inherent uncertainty of axisymmetric RANS simulations in the present case of large flow separations within the first nozzle.

Further measurements were performed with screens. Two screens made from stainless steel are employed. The open ratios of the two screens are different, one mesh size is $0.8 \times 2 \mathrm{~mm}$ and the other is $0.63 \times 2 \mathrm{~mm}$. The screen with large size was always placed ahead of the other when two screens were used; otherwise, only the screen with large mesh size was employed. Two aluminum rings were used to clamp the screen. 


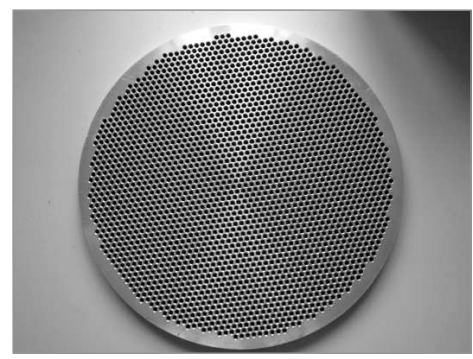

(a)

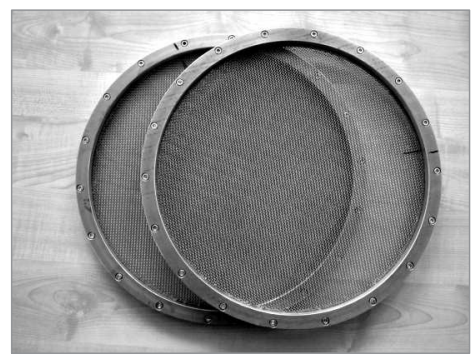

(b)

Figure 13 Perforated plate $(a)$ and screens $(b)$ tested in the settling chamber

The perforated plate and the screen are shown in Fig. 13. These screens were tested in the settling chamber by replacing the perforated plates at the 3rd and 4th positions independently. The influence of the settling chamber configurations to the test section flow are compared in Fig. 14. Figure 14a shows the Pitot signals for different numbers of perforated plates, while Fig. $14 b$ displays the Pitot signals for various screen configurations. Here, only the 3rd and 4 th positions have been replaced with screens. Either replacing one plate or both plates was tested. It is found that the configuration of four plates installed in settling chamber appears better than other cases. Note that it may be worth testing the effect of a fifth device in the future.

From these tests of different configurations of the settling chamber, it is apparent that the screens have little effect on the flow quality improvement, while the plates exhibit a larger influence. According to the numerical study of [3], the initial flow in the settling chamber downstream of the separated first nozzle is strongly inhomogeneous with a core flow at high velocity. This is also seen in Fig. 12. Hence, the purpose of the settling chamber inserts is to damp out the flow inhomogeneity by providing total pressure losses. The perforated plate has a larger thickness and smaller open ratio than the screens, resulting in a larger pressure drop. However, the described measurements can only partially indicate the total fluctuation of the flow because of the low dynamic response of the pressure sensor. Therefore, a 7 degree half-angle sharp cone model with zero angle of attack was also utilized to check the ability of the settling chamber inserts to damp the flow disturbance. The flow condition of the storage tube was kept constant in these tests, e.g., the storage pressure was 10 bar and the temperature was $430 \mathrm{~K}$. The spectra of two sensors, namely, sensors 1 and 9, are shown in Fig. 6. Figure 15 displays the amplitude spectrum of the measured pressure fluctuations. The amplitude of sensor 9 is larger than that of sensor 1 , probably, because of the growth of boundary layer disturbances along the cone as well as due to variations of flow disturbances along the test section by the effect 

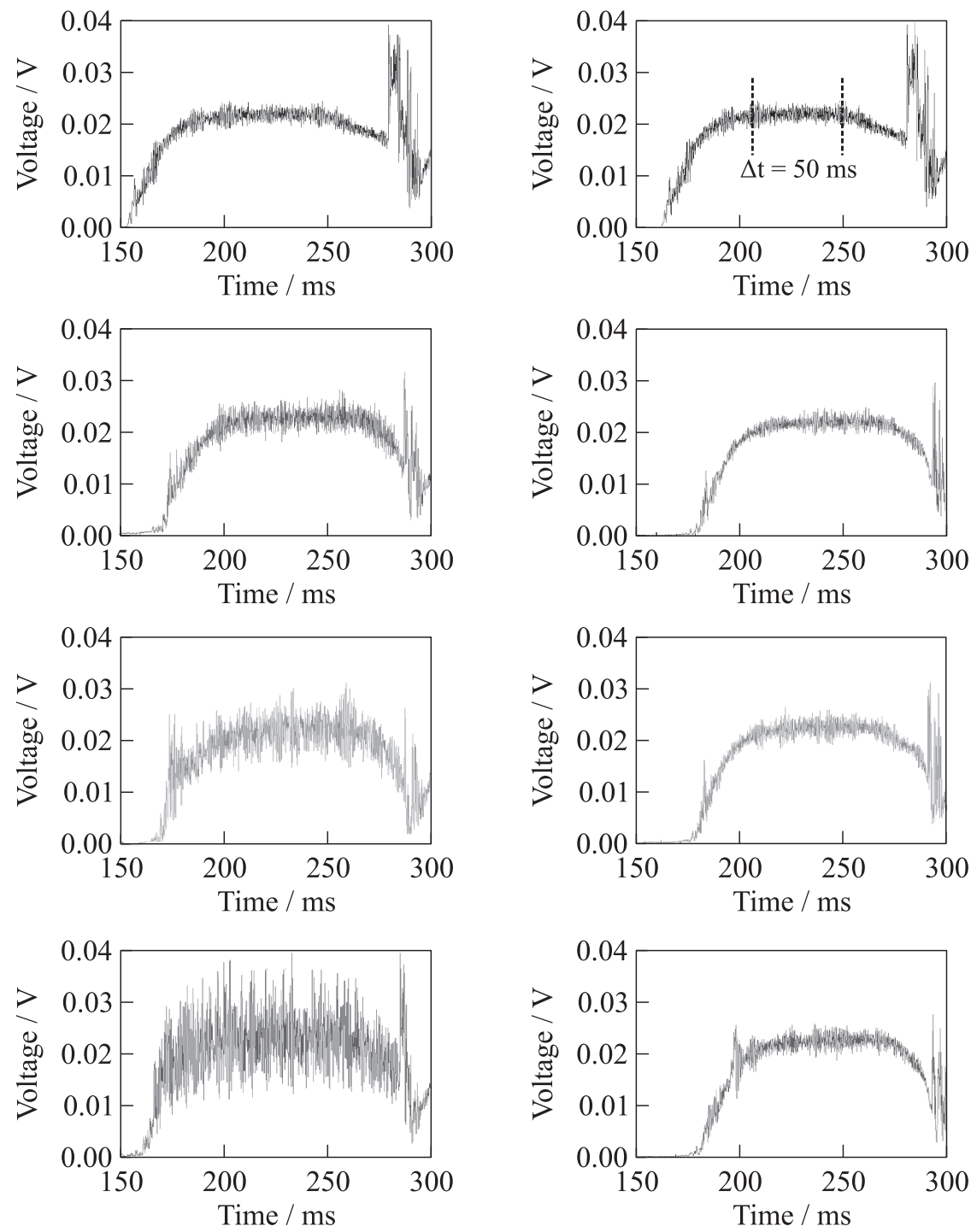

(a)

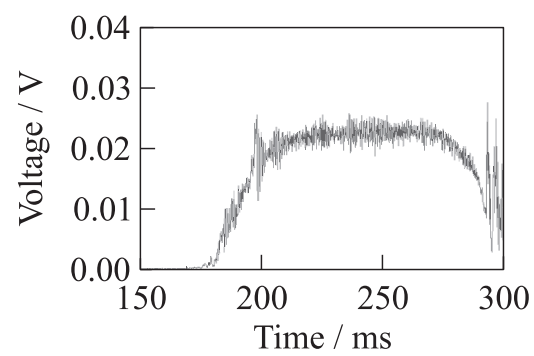

(b)

Figure 14 Effect of perforated plates and screens of Pitot pressure in the test section at $x=60 \mathrm{~mm}$ and $z=0 \mathrm{~mm}:(a)$ time trace signals of Pitot pressure for different numbers of perforated plates (from top to bottom: 4 plates; 3 plates; 2 plates; and 1 plate); and (b) time trace signals of Pitot pressure for combinations of perforated plates and screens (from top to bottom: 4 plates; 3rd plate replaced by a screen; 4th plate replaced by a screen; and 3rd and 4th plates replaced by two screens) 


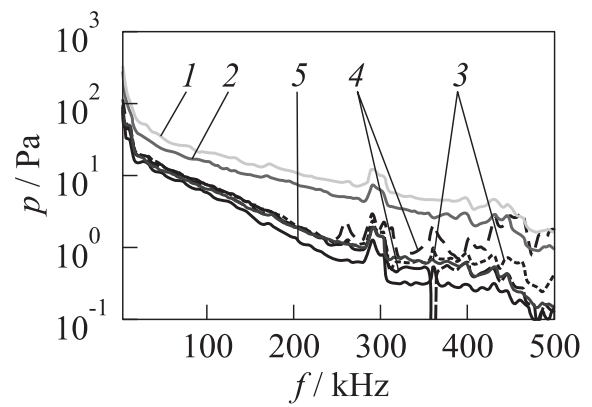

(a)

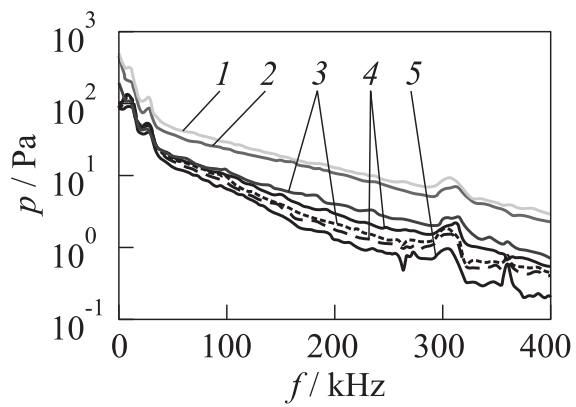

(b)

Figure 15 Spectra comparison of sensors $1(a)$ and $2(b)$ of different inserts in settling chamber: $1-0$ plates; $2-1$ plate; $3-2$ plates; $4-3$ plates; $5-4$ plates; solid curves -0 screens; dashed curves -1 screen; and dotted curves -2 screens

shivering waves. For less than two perforated plates in the settling chamber, one observes a distinct increase of the amplitude of the signals, which may be a sign of transition on the cone. Nevertheless, the flow case of four perforated plates installed in the settling chamber displays the lowest amplitude, which agrees well with the Pitot probe measurement in Fig. 14.

\subsection{Test Section Measurement}

First, the repeatability of Pitot signals was studied by placing the Pitot rake in the middle of the test section and running the wind tunnel three times with the same flow conditions in the storage tube. Based on the Pitot pressure, the Mach number can be evaluated based on the well-known Rayleigh-Pitot formula. The Mach numbers obtained are shown in Fig. 16. The data indicate that the Pitot probe measurements in the test section are well repeatable.

Detailed line traverses are presented in Fig. 17, at $x=60,100,150$, and $200 \mathrm{~mm}$ (see Fig. 4). The initial pressure in the storage tube was kept at 10 bar and the temperature was set to $300 \mathrm{~K}$. The obtained Mach number data are compared with the RANS calculations of the measured nozzle contour which is performed in the same way as in [3], as shown in Fig. 17. Compared with the numerical result, the experimental measurement is in good agreement although small deviations close to the tunnel axis are observed at $x=150$ (see Fig. 17c) and $200 \mathrm{~mm}$ (see Fig. 17d). The amount of these deviations is around $0.04 \mathrm{in}$ the Mach number. Note that the nonlinearity of the pressure sensor results in possible measurement errors of \pm 0.025 .

In further measurements, the cross section at $x=100 \mathrm{~mm}$ is checked by traversing the Pitot rake in both $z$ - (vertical) and $y$ - (horizontal) directions as 


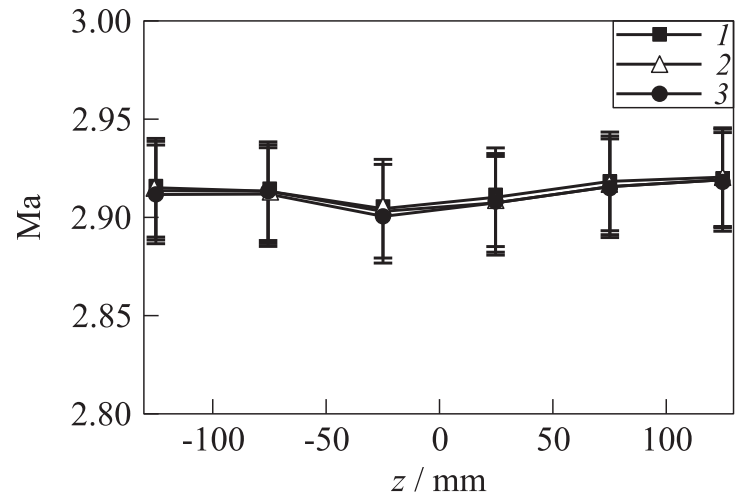

Figure 16 Flow repeatability validation in test section: 1 - repeat-1; 2 - repeat-2; and 3 - repeat-3

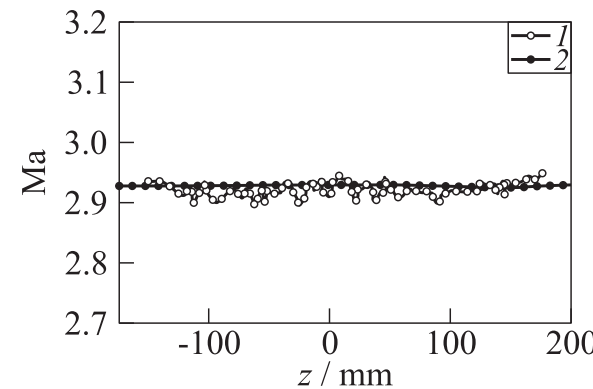

(a)

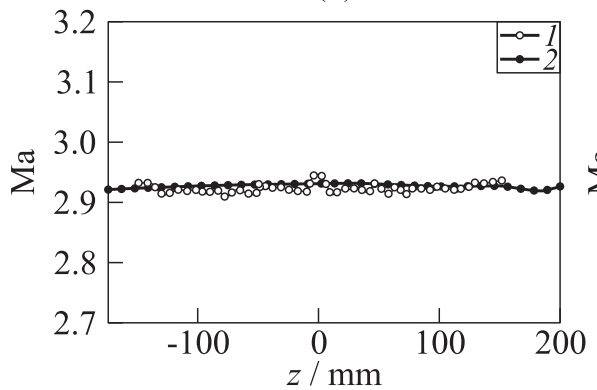

(c)

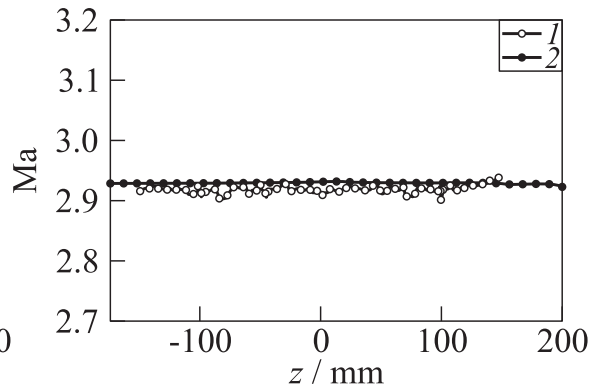

(b)

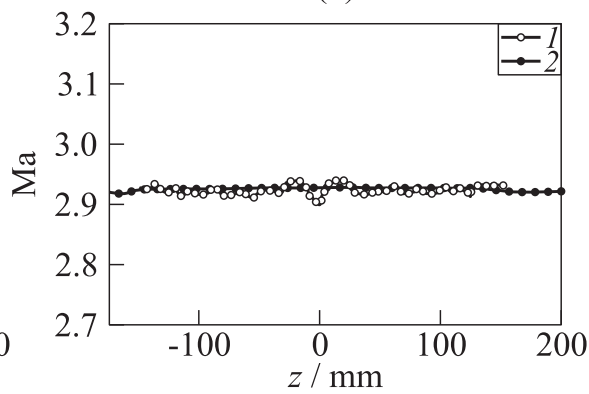

(d)

Figure 17 Mach number comparisons between the measurement (1) and RANS simulations (2): (a) $x=60 \mathrm{~mm}$; (b) $100 ;(c) 150$; and $(d) x=200 \mathrm{~mm}$ 


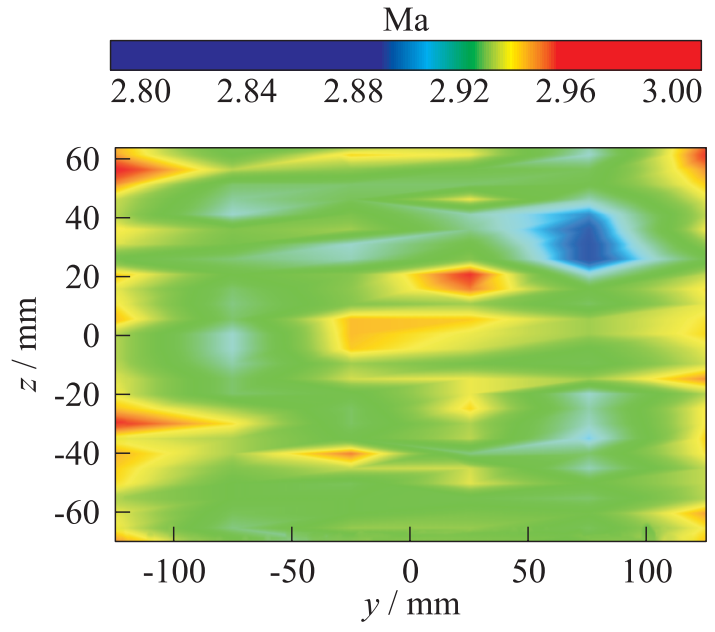

Figure 18 Mach number contours from Pitot measurement obtained at $x=100 \mathrm{~mm}$

shown in Fig. 18. The Mach contours in the cross section display a rather homogeneous flow despite some Mach scatters exist. Note again that the uncertainty of the measurement due to the nonlinearity of the pressure sensor is \pm 0.025 , which almost covers the Mach number scatter observed in Fig. 18.

The 7 degree half-angle sharp cone model installed with PCB sensors was utilized to assess the pressure fluctuations in more detail. Because the flow close to the axis appears somewhat disturbed as shown above, the model was placed $108 \mathrm{~mm}$ above the tunnel axis. The temperature of the flow in the storage tube was set to $300 \mathrm{~K}$, and the storage tube pressure varied from 8 to 13 bar. The spectra of the pressure fluctuations for the sensors along the cone as described in subsection 3.3 are compared in Fig. 19. Note that only two sensors of group 1 (G1) were active during the measurements. At the freestream Reynolds number of $4.77 \cdot 10^{6}$ (see Fig. 19a), the fluctuations of G2 are significantly above the other sensors. This is explained by transition of the laminar boundary layer to the turbulent flow. As the unit Reynolds number increases, the transition location moves forward. At values of $6.56 \cdot 10^{6}$ (see Fig. 19d), the spectra of G1 group are very close to the G2 group. This indicates transition Reynolds numbers of around $1.7 \cdot 10^{6}$ for almost an adiabatic wall. At the largest Reynolds number of $7.75 \cdot 10^{6}$ (see Fig. 19f), all sensors indicate turbulent boundary layer flow. While one could observe transition as a function of Reynolds number from these data, still it was not possible to identify and characterize the primary instabilities of the transition process. However, the spectra display weak bumps at frequencies between 10 to $50 \mathrm{kHz}$. These 


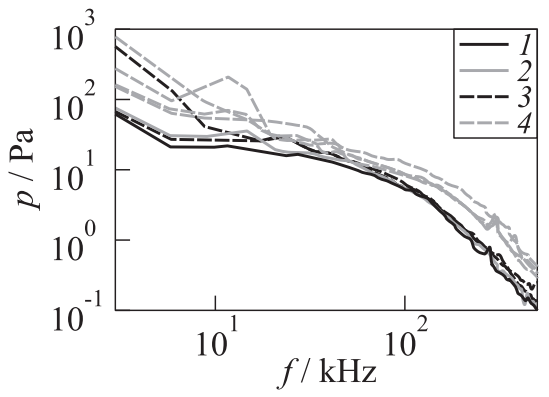

(a)

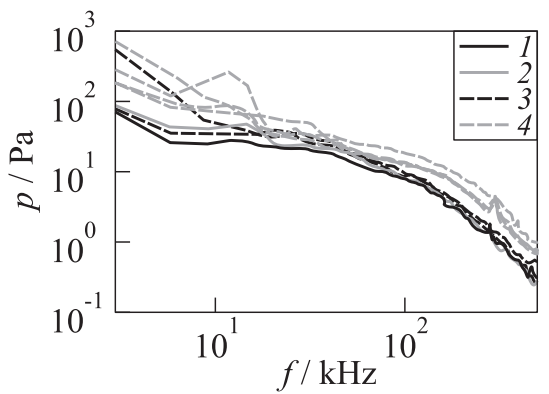

(c)

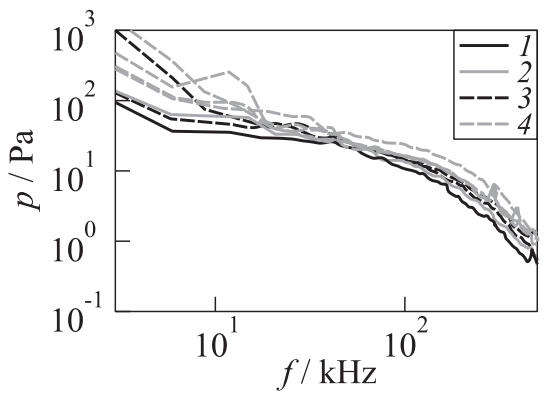

(e)

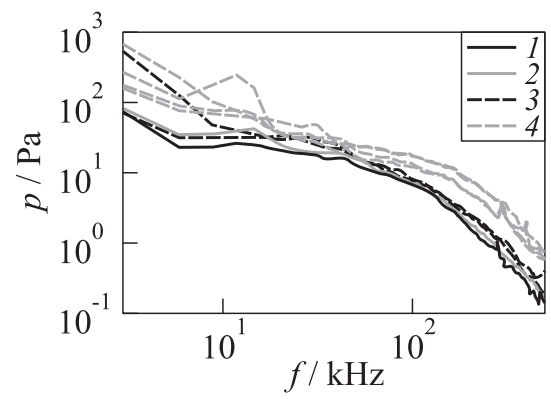

(b)

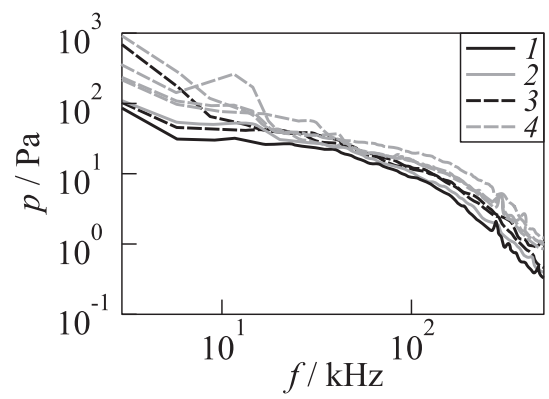

(d)

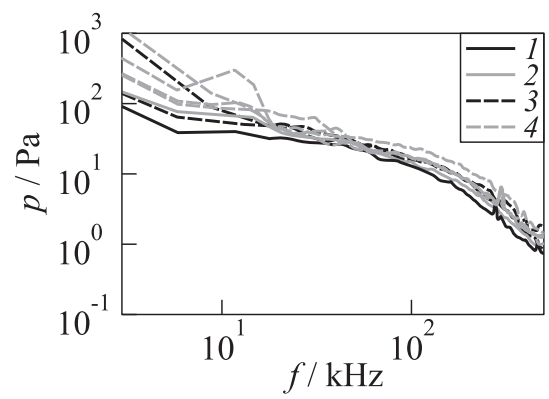

$(f)$

Figure 19 Spectra comparison of PCB sensors $(1-$ sensor 1 ; 2 - sensor 2 ; 3 - G1; and $4-$ G2) in sharp cone model: $(a) R_{\text {unit }}=4.77 \cdot 10^{6} ;(b) 5.37 \cdot 10^{6} ;(c) 5.96 \cdot 10^{6}$; (d) $6.56 \cdot 10^{6} ;(e) 7.15 \cdot 10^{6} ;$ and $(f) \operatorname{Re}_{\text {unit }}=7.75 \cdot 10^{6}$

bumps at low frequencies may be related to the first-mode instabilities of supersonic boundary layer flow. Further studies will be needed to investigate the low-frequency range with Kulite sensors that can resolve frequencies down to $10 \mathrm{kHz}$ and below. Also, hot wire measurements will be employed to characterize instabilities. 


\section{CONCLUDING REMARKS}

The present work displays the comprehensive investigation of a newly designed tandem nozzle Mach 3 wind tunnel at the Institute of Fluid Mechanics, Technische Universität Braunschweig. The flow development along the tunnel is characterized by installing pressure sensors at different locations. The analysis shows that the measured pressure distribution of the tunnel agrees moderately well with the numerical design. Detailed studies of the settling chamber show the favourable effects of perforated plates to damp out flow disturbances. The pressure drop in the settling chamber shows some differences compared with previous design calculation using the RANS equations. The temperature distribution for heated operations was measured by thermocouples. The results display that the effects of the temperature stratification in the storage tube are also found at the entrance to the second nozzle. The test section flow was measured by traversing a Pitot rake, and the results show good agreement with the RANS simulation. Unsteady pressure fluctuations are measured on an instrumented sharp cone model at zero angle of attack. Transition Reynolds numbers of $1.7 \cdot 10^{6}$ are found for the 7 degree sharp cone and the current wind tunnel configuration. The flow quality in the test section and boundary layer transition need further study. Therefore, hot wire measurements and the application of LDI (Laser differential interferometer) are planned in the freestream and in the boundary layer flow. In conclusion, the present works demonstrate the potentials and feasibility to extend the operation range of high-speed wind tunnels by using the tandem nozzle concept.

\section{REFERENCES}

1. Ludwieg, H. 1955. Der Rohrwindkanal. Zeitschrift für Flugwissenschaften 3:206216.

2. Koppenwallner, G., R. Müller-Eigner, and H. Friehmelt. 1993. HHK HochschulHyperschall-Kanal: Ein "Low cost" Windkanal für Forschung und Ausbildung. DGLR Jahrbuch. Band 2.

3. Wu, J. and R. Radespiel. 2013. Tandem nozzle supersonic wind tunnel design. Int. J. Eng. Syst. Modelling Simulation 5:8-18.

4. Estorf, M., T. Wolf, and R. Radespiel. 2005. Experimental and numerical investigation on the operation of the Hypersonic Ludwieg Tube Braunschweig. 5th Symposium on Aerodynamics for Space Vehicles Proceedings. 8-11.

5. Schrijer, F. F. J. 2010. Experimental investigation of reentry aerodynamic phenomena. Oisterwijk, The Netherlands. Ph.D. Thesis.

6. Anderson, J. D. 2003. Modern compressible flow: With historical perspective. 3rd ed. Singapore: McGraw-Hill. 167-175. 
7. Munoz, F., D. Heitmann, and R. Radespiel. 2012. Instability modes in boundarylayers of an inclined cone at Mach 6. AIAA Paper No. 2012-2823.

8. Estorf, M., R. Radespiel, S. P. Schneider, H. Johnson, and S. Hein. 2008. Surfacepressure measurements of second-mode instability in quiet hypersonic flow. AIAA Paper No. 2008-1153.

9. Heitmann, D., R. Radespiel, and H. Knauss. 2008. Experimental study of Mach 6 boundary-layer response to laser generated disturbances. AIAA Paper No. 20113876.

10. Groth, J., and A.V. Johansson. 1988. Turbulence reduction by screens. J. Fluid Mech. 197:139-155. 